\title{
Quantum Phase Transitions in the Interacting Boson Model: Integrability, Level Repulsion, and Level Crossing
}

\author{
J. M. Arias, ${ }^{1}$ J. Dukelsky, ${ }^{2}$ and J. E. García-Ramos ${ }^{3}$ \\ ${ }^{1}$ Departamento de Física Atómica, Molecular y Nuclear, Facultad de Física, Universidad de Sevilla, \\ Apartado 1065, 41080 Sevilla, Spain \\ ${ }^{2}$ Instituto de Estructura de la Materia, CSIC, Serrano 123, 28006 Madrid, Spain \\ ${ }^{3}$ Departamento de Física Aplicada, Universidad de Huelva, 21071 Huelva, Spain
}

(Received 20 June 2003; published 14 October 2003)

\begin{abstract}
We study the quantum phase transition mechanisms that arise in the interacting boson model. We show that the second-order nature of the phase transition from $U(5)$ to $O(6)$ may be attributed to quantum integrability, whereas all the first-order phase transitions of the model are due to level repulsion with one singular point of level crossing. We propose a model Hamiltonian with a true firstorder phase transition for finite systems due to level crossings.
\end{abstract}

Quantum phase transitions (QPTs) have attracted great attention from the theoretical and experimental communities in recent years. Experiments on high- $T_{c}$ superconductors, on quantum-Hall systems, on Bose or Fermi dilute gases in different trap geometries, and on nuclei close to a critical point challenge theoreticians to develop reliable approaches with which to describe the critical properties of these systems.

A QPT describes a structural change in the properties of the ground state of the system associated with the variation of a control parameter (the temperature being the usual one in classical phase transitions). This parameter might be the level of doping in high- $T_{c}$ superconductors, the magnetic field in quantum-Hall systems, the scattering length in dilute gases, the nucleon number in nuclear phase transitions, etc.

The phase diagram of the interacting boson model (IBM) [1] of nuclei has been studied at the mean-field level [2,3], using both catastrophe theory [4] and the Landau theory of phase transitions [5]. Both treatments show that the model displays first-order phase transitions from spherical to deformed shapes and from oblate to prolate deformed shapes, with an exceptional point of an isolated second-order phase transition. In this work, we study in detail the phase transitions associated with the IBM.

The IBM is a phenomenological model of nuclear structure with a deep connection to the underlying microscopic shell model. In this model, which has been very successful in describing nuclear spectroscopic properties of collective nuclei, pairs of correlated nucleons of angular momentum $L=0$ and $L=2$ are represented by $s$ and $d$ bosons, respectively. In its simplest version the model does not distinguish between protons and neutrons and has an underlying group structure based on the dynamical group $\mathrm{U}(6)$.

The most general IBM Hamiltonian can be written in terms of six free parameters, as a linear combination of the linear and quadratic Casimir operators of $\mathrm{U}(5)$ and the quadratic Casimir operators of $\mathrm{O}(6), \mathrm{SU}(3), \mathrm{O}(5)$, and $\mathrm{O}(3)$. A convenient and frequently used form of the IBM Hamiltonian that keeps all the main ingredients related to the structure of the ground state is the consistent- $Q$ Hamiltonian [6]

$$
H=x n_{d}+\frac{x-1}{N} Q^{\chi} \cdot Q^{\chi},
$$

where $n_{d}=\sum_{\mu} d_{\mu}^{\dagger} d_{\mu}, \quad Q_{\mu}^{\chi}=\left[d^{\dagger} \tilde{s}+s^{\dagger} \tilde{d}\right]_{\mu}^{2}+\chi\left[d^{\dagger} \tilde{d}\right]_{\mu}^{2}$, and $N$ is the total number of bosons, which is equal to the number of nucleon pairs in the valence space.

One of the most important features of the IBM is the existence of four distinct dynamical symmetries (DS), each representing a well-defined phase of nuclear collective motion. A quantum system has a DS if the Hamiltonian can be expressed as a linear combination of the Casimir operators of a subgroup chain of the dynamical group. The four dynamical symmetries are the following: the $\mathrm{U}(5)$ symmetry for spherical vibrational nuclei $(x=$ 1), the $\mathrm{SU}(3)$ symmetry for prolate deformed nuclei $(x=$ $0, \chi=-\sqrt{7} / 2)$, the $\mathrm{O}(6)$ symmetry for $\gamma$ unstable deformed nuclei $(x=0, \chi=0)$, and the $\overline{\mathrm{SU}(3)}$ symmetry for oblate deformed nuclei $(x=0, \chi=\sqrt{7} / 2)$.

The symmetries of the IBM and the transitions between them are illustrated in the extended Casten triangle [7] of Fig. 1, along with the corresponding values of the parameters $x$ and $\chi$. Distinct phases associated with three "shapes," denoted S for spherical, P for prolate deformed, and $\mathrm{O}$ for oblate deformed, can be seen in the figure. The three different shape phases are separated by two lines of first-order phase transitions, $x=0.8$ and $\chi=0$, respectively, with the exception of a triple point [5] at $x=0.8$ and $\chi=0$, which is represented by a solid square and for which the phase transition is second order. Recently, two effective models based on the Bohr Hamiltonian, called $X(5)$ and $E(5)$, have been proposed to describe the physics 


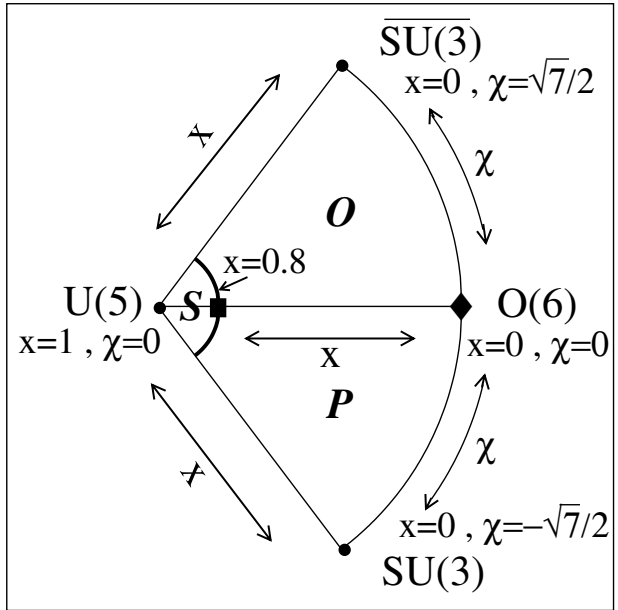

FIG. 1. Phase diagram of the interacting boson model in the phase space of the control parameters $x$ and $\chi$. S, O, and $\mathrm{P}$ stand for the spherical, oblate, and prolate phases, respectively.

at the critical points along the $\mathrm{U}(5)-\mathrm{SU}(3)$ and $\mathrm{U}(5)-$ $\mathrm{O}(6)$ lines, respectively [8].

The aim of this Letter is to go beyond the mean-field description of the phase diagram in Fig. 1. We will show that the first-order QPTs arise due to level repulsion between two states with different properties, as was earlier recognized in [9]. However, there are two exceptional singular critical points, represented by the bold square and the bold diamond in Fig. 1, for which the mechanism triggering the phase transition is completely different.

We begin with a discussion of the transition from $\mathrm{O}(6)$ to $\mathrm{U}(5)$. It is described within the Hamiltonian (1) by setting $\chi=0$ and varying $x$ from 0 to 1 . Earlier investigations of the properties of the level spacings along this leg of the Casten triangle showed a Poisson distribution. This indicates that the model is integrable along this leg [10], even though it is not characterized by a global dynamical symmetry.

Quantum integrability requires the existence of a complete set of mutually commuting Hermitian operators. These operators are the integrals of motion or quantum invariants and their eigenvalues are the conserved quantities that completely label a unique basis of common eigenstates. A DS is a particular class of integrable model in which each one of the Casimir operators in the subgroup decomposition chain is a quantum invariant. But there are also other integrable and exactly solvable models that have played important roles in the understanding of quantum many-body systems. Examples include the Heisenberg and Hubbard models, both of which are solvable by the Bethe ansatz. The Hamiltonian (1) for $\chi=0$ is a particular case of a general class of pairing models that have been shown to be quantum integrable [11]. The quantum invariants for boson pairing Hamiltonians and the corresponding exact solutions were discussed in Ref. [12]. Here we will specify the form of the quantum invariants in the $\mathrm{O}(6)-\mathrm{U}(5)$ transition. Since we are interested only in properties of the ground state, we focus our treatment on the case in which all bosons are paired to zero angular momentum.

We first note that this transition is embedded in the decomposition $\mathrm{U}(6) \supset \cdots \supset \mathrm{O}(5) \supset \mathrm{O}(3) \supset \mathrm{O}(2)$, where a missing parameter dependent "symmetry" or quantum invariant commuting with the Casimir operators of all the groups in the chain is responsible for the integrability of the model.

As noted in Refs. [10,13], the Hamiltonian itself, interpolating between the Casimir operators of U(5) and $\mathrm{O}(6)$, constitutes the additional independent quantum invariant ensuring the integrability of the system.

The eigenvalues of the Hamiltonian (1) for $\chi=0$ are [12]

$$
E=\frac{20(x-1)}{N} \sum_{\alpha=1}^{N / 2} \frac{1}{2-e_{\alpha}},
$$

where the pair energies $e_{\alpha}$ are the solutions of the coupled set of nonlinear Richardson equations

$$
\frac{x N}{2(1-x)}-\frac{1}{e_{\alpha}}+\frac{5}{2-e_{\alpha}}-4 \sum_{\beta(\neq \alpha)} \frac{1}{e_{\alpha}-e_{\beta}}=0 .
$$

The Hamiltonian eigenvalues (2) are analytic functions of the control parameter $x$. Therefore, in the absence of level crossings at the ground-state level, a first-order phase transition is precluded. Level crossings can occur only for integrable models with more than one parameter dependent integral of motion, an example being the $s d g$ boson model [14].

Because of the exact solvability of the model, we are able to obtain exact results numerically for a large number of bosons. In Fig. 2, we show results for 1000 bosons and compare them with the mean-field solution. In the left
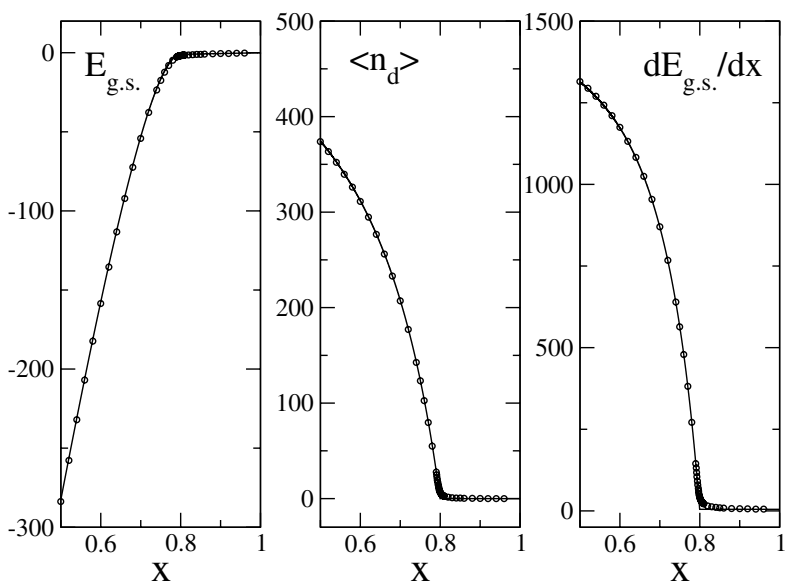

FIG. 2. Transition from $\mathrm{O}(6)$ to $\mathrm{U}(5)$. The ground-state energy (left panel), expectation value of the $n_{d}$ (central panel), and derivative of the ground-state energy (right panel) are presented as a function of the control parameter $x$ for a system with 1000 bosons. The exact results are represented by circles and the mean field results by a full line. 
panel, we plot the ground-state energy versus the control parameter $x$; in the central panel, the expectation value of the number of $d$ bosons $\left\langle n_{d}\right\rangle$ is presented as a function of $x$; in the right panel, we plot the derivative of the energy versus $x$. Because of the exact solvability of the model, we can make use of the Hellmann-Feynman theorem to express the first derivative of the energy as the expectation value of the derivative of the Hamiltonian (1) with respect to $x$, i.e., $(\partial H / \partial x)=n_{d}+(1 / N) Q^{0} \cdot Q^{0}$. Both operators, $n_{d}$ and $(\partial H / \partial x)$, are zero in one phase (disordered) and different from zero in the other phase (ordered), fulfilling the requirements for being order parameters. Both expectation values are continuous at the transition point satisfying both the Landau and Ehrenfest definitions of a second-order phase transition.

We will now discuss the phenomenon of level repulsion for nonintegrable systems. For an arbitrary finite value of $\chi$, we select a value of $x=x_{0}$ in the vicinity of which we can use nondegenerate perturbation theory. Defining $x^{\prime}=$ $x-x_{0}$, we can rewrite the Hamiltonian as $H=x_{0} n_{d}+$ $\left[\left(x_{0}-1\right) / N\right] Q^{\chi} \cdot Q^{\chi}+x^{\prime}\left(n_{d}+\frac{1}{N} Q^{\chi} \cdot Q^{\chi}\right)=H_{0}+x^{\prime} V$. The first and second derivatives of the Hamiltonian eigenvalues in perturbation theory are [15]:

$$
\begin{gathered}
\frac{\partial E_{n}}{\partial x^{\prime}}=\frac{\partial E_{n}}{\partial x}=V_{n n}\left(x_{0}\right), \\
\frac{\partial^{2} E_{n}}{\partial x^{\prime 2}}=\frac{\partial^{2} E_{n}}{\partial x^{2}}=2 \sum_{m \neq n} \frac{V_{n m}^{2}\left(x_{0}\right)}{E_{n}\left(x_{0}\right)-E_{m}\left(x_{0}\right)} .
\end{gathered}
$$

Assuming that there is a pair of energy levels that get very close in energy, the energy gap between them is $\Delta(x)=E_{2}(x)-E_{1}(x)$. Neglecting the contribution of all other states, perturbation theory for the gap derivative gives

$$
\frac{\partial \Delta}{\partial x}=V_{22}-V_{11}, \quad \frac{\partial^{2} \Delta}{\partial x^{2}}=\frac{4 V_{12}^{2}}{\Delta} .
$$

We can interpret $E_{1}$ and $E_{2}$ as the positions of two fictitious classical particles in a one-dimensional space. Then (6) are the equations for their relative velocity and the force between them. Clearly, if the interaction matrix element $V_{12}$ remains finite as $\Delta \rightarrow 0$, there is an infinite repulsion and this prevents the particles from colliding. This is a pictorial view of the phenomenon of level repulsion for nonintegrable Hamiltonians. On the other hand, if there is an additional conserved quantity and both levels have different symmetries, then $V_{12}$ is strictly zero and the two levels can cross. In what follows, we discuss the application of these ideas first to the $\mathrm{U}(5)$ to $\mathrm{SU}(3)$ transition and then to the $\overline{\mathrm{SU}(3)}$ to $\mathrm{SU}(3)$ transition.

In Fig. 3, we show in the left panel the ten lowest $0^{+}$ states for a system of 50 bosons as a function of $x$ along the $\mathrm{U}(5)$ to $\mathrm{SU}(3)$ transition. There are several cases in which two $0^{+}$levels get very close in energy but, as just discussed, the closer they get the larger the level repulsion between them is. In the right panels, we magnify two of the cases of closest approach to show that indeed the

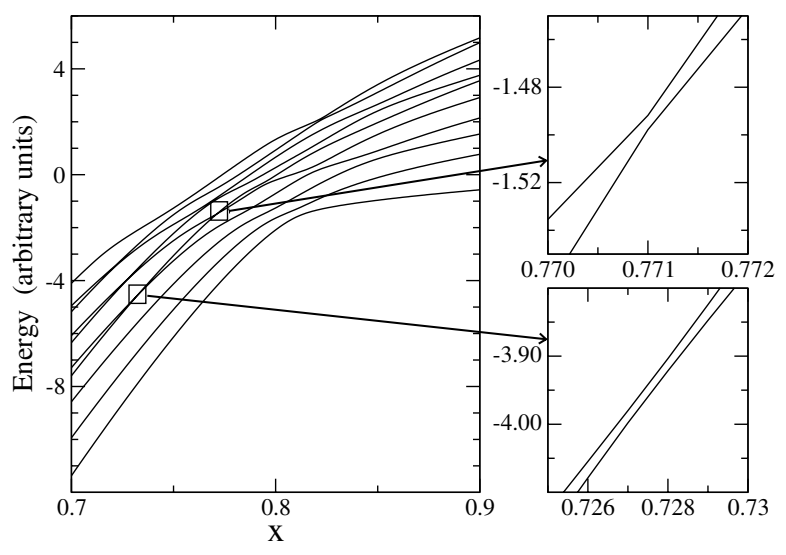

FIG. 3. Ten lowest $0^{+}$states in the transition from $\mathrm{U}(5)$ to $\mathrm{SU}(3)$ for $N=50$ bosons as a function of $x$. On the right panels we show in an enlarged scale two examples of level repulsion.

levels repel and do not cross. In particular, there is a smooth crossover in the ground-state energy at the point of closest approach with the first excited state. The dynamics of these two levels is well described by Eq. (6) with finite matrix elements $V_{i j}$. The crossover will change gradually as the number of bosons increases towards a nonanalytic point in the thermodynamic limit, defining a first-order phase transition. From the above, we conclude that level repulsion is indeed responsible for the firstorder phase transition in this case [9].

Now we turn to the third leg of the triangle $(x=0)$, the transition from deformed oblate [SU(3) shapes, $\chi=$ $\sqrt{7} / 2$ ] to deformed prolate [SU(3) shapes, $\chi=-\sqrt{7} / 2]$ shapes, passing through the $\gamma$-unstable $\mathrm{O}(6)$ case with $\chi=0$. In this case, the first-order phase transition takes place precisely at the $\mathrm{O}(6)$ dynamical symmetry [7]. At this critical point, the $0^{+}$states can be classified with the $\tau$ quantum number of an $\mathrm{O}(5)$ symmetry. As discussed above, the realization of this new symmetry allows the crossing of levels with different quantum numbers. The Hamiltonian (1) at this point is

$$
H=-\frac{1}{N} Q^{0} \cdot Q^{0}=\frac{1}{2 N}\left(C_{2}[\mathrm{O}(6)]-C_{2}[\mathrm{O}(5)]\right),
$$

with eigenvalues $E_{\sigma \tau}=[-\sigma(\sigma+4)+\tau(\tau+3)] / 2 N$. The lowest $\mathrm{O}(6)$ band corresponds to $\sigma=N$ and $\tau=$ $0,3, \ldots$ In the large $N$ limit, the $\mathrm{O}(5)$ states within the ground-state $\mathrm{O}(6)$ band will be degenerate, since the energy spacings go as $N^{-1}$. According to the above analysis, in the thermodynamic limit an infinite set of $0^{+}$states with different seniority quantum numbers $\tau$ cross. The crossing is allowed because of the $\mathrm{O}(5)$ symmetry at the critical point, implying that the interaction matrix elements in (6) are zero.

As just noted, the exact degeneracy in the levels of the ground-state band at $x=0$ and $\chi=0$ occurs only in the large- $N$ or thermodynamic limit. To better visualize this QPT in the context of finite systems, we therefore consider a Hamiltonian similar to that in (1), but with an 


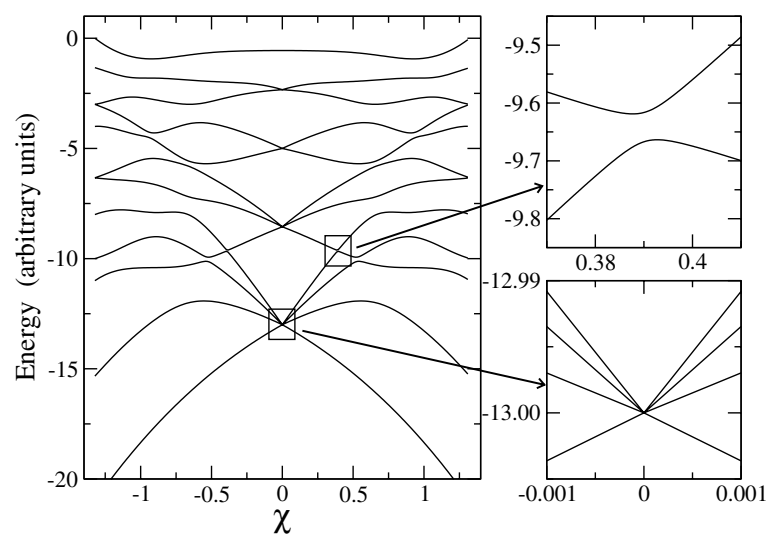

FIG. 4. Transition from $\overline{\mathrm{SU}(3)}$ to $\mathrm{SU}(3)$ through the $\mathrm{O}(6)$ limit. All $0^{+}$eigenstates of the Hamiltonian (8) for a system with $N=9$ bosons are represented as a function of the control parameter $\chi$. The right panels show in a larger scale the level repulsion of two close levels at $\chi \neq 0$ (right-top panel) and the level crossing at $\chi=0$ (right-bottom panel).

additional term that cancels the $\mathrm{O}(5)$ term in it that produces energy spacings within the ground-state band for finite $N$ [see Eq. (7)]. With this in mind, we consider the following Hamiltonian associated with the $x=0$ transition leg:

$$
H=-\frac{1}{N}\left[Q^{\chi} \cdot Q^{\chi}+\left(\frac{1}{2}-\frac{|\chi|}{\sqrt{7}}\right) C_{2}[\mathrm{O}(5)]\right] .
$$

For $\chi=-(\sqrt{7} / 2)$, the Hamiltonian (8) is in the $\mathrm{SU}(3)$ limit; for $\chi=-(\sqrt{7} / 2)$, it is in the $\overline{\mathrm{SU}(3)}$ limit; and for $\chi=0$ it is proportional to the quadratic Casimir operator of the $\mathrm{O}(6)$ group. The second term in the modified Hamiltonian (8) thus cancels out the $\mathrm{O}(5)$ breaking of the degeneracies in the ground-state band that occurs for finite $N$.

In Fig. 4, we show all of the $0^{+}$eigenstates of the Hamiltonian (8) for a system with $N=9$ bosons as a function of $\chi$. At $\chi=0$, the four levels that correspond to the ground-state band with $\sigma=9$ cross. This crossing gives rise to a first-order QPT for a "finite" system. This is the unique point along the $x=0$ leg at which level crossings are allowed due to the realization of the $\mathrm{O}(5)$ symmetry. Away from this point the mechanism of level repulsion sets in. In the upper right panel of Fig. 4, we show in an enlarged scale an apparent level crossing at $\chi \neq 0$. The mechanism of level repulsion predicted in Eq. (6) is numerically verified. On the contrary at $\chi=0$, the realization of the $\mathrm{O}(5)$ symmetry allows the crossing of levels with different $\tau$ quantum numbers, as shown in the lower right panel.

In summary, there are two singular points of QPTs in the IBM phase diagram. The first is in the transition from $\mathrm{U}(5)$ to $\mathrm{O}(6)$ and corresponds to a second-order QPT due to the integrable nature of the Hamiltonian along this line, which ensures that the ground-state energy is an analytic function of the control parameter. The second singular point is located at the QPT from $\mathrm{SU}(3)$ to $\mathrm{SU}(3)$, precisely at the $\mathrm{O}(6)$ critical symmetry. In the thermodynamic limit, this QPT is associated with the crossing of an infinite number of $0^{+}$states. By adding to the Hamiltonian (7) a term proportional to the $\mathrm{O}(5)$ Casimir operator, we were able to realize this QPT for a finite system. This is an unusual, but especially interesting, case to study further, since most phase transitions take place in the thermodynamic limit. Therefore, the model described by the Hamiltonian (8) may be useful to explore the universal properties of first-order phase transitions.

These two isolated critical points represent exceptional points in the phase diagram of the IBM. Along the lines separating the spherical, prolate, and oblate phases, the mechanism underlying the first-order phase transitions is level repulsion between the ground state and the first excited state. For finite $N$, this mechanism is reflected by a soft crossover, but in the large $N$ limit the model develops a singularity in the derivative of the gap consistent with a first-order phase transition. This conclusion is also valid for more general IBM Hamiltonians, since level repulsion is a universal phenomenon associated with quantum nonintegrability.

This work was supported in part by the Spanish DGI under Projects No. BFM2002-03315 and No. BFM20001320-C02-02. We acknowledge useful discussions with S. Pittel, P. Van Isacker, J. Jolie, and F. Iachello.

[1] F. Iachello and A. Arima, The Interacting Boson Model (Cambridge University Press, Cambridge, 1987).

[2] A. E. L. Dieperink, O. Scholten, and F. Iachello, Phys. Rev. Lett. 44, 1747 (1980).

[3] D. H. Feng, R. Gilmore, and S. R. Deans, Phys. Rev. C 23, 1254 (1981).

[4] E. López-Moreno and O. Castaños, Phys. Rev. C 54, 2374 (1996).

[5] J. Jolie et al., Phys. Rev. Lett. 89, 182502 (2002).

[6] R. F. Casten and D. D. Warner, Rev. Mod. Phys. 60, 389 (1988).

[7] J. Jolie, R. F. Casten, P. von Brentano, and V. Werner, Phys. Rev. Lett. 87, 162501 (2001).

[8] F. Iachello, Phys. Rev. Lett. 85, 3580 (2000); 87, 052502 (2001).

[9] N.V. Zamfir, P. von Brentano, R. F. Casten, and J. Jolie, Phys. Rev. C 66, 021304 (2002).

[10] Y. Alhassid and N. Whelan, Phys. Rev. Lett. 67, 816 (1991).

[11] J. Dukelsky. C. Esebbag, and P. Schuck, Phys. Rev. Lett. 87, 066403 (2001).

[12] J. Dukelsky and S. Pittel, Phys. Rev. Lett. 86, 4791 (2001)

[13] P. Cejnar and J. Jolie, Phys. Rev. E 58, 387 (1998).

[14] J. Dukelsky, J. M. Arias, J. E. García-Ramos, and S. Pittel, nucl-th/0310031.

[15] E. A. Yuzbashyan, B. L. Altshuler, and B.S. Shastry, J. Phys. A 35, 7525 (2002). 